Rejane R D Casagrande'

Antonio C Pastorino'

Renata G L Souza'

Cláudio Leone'

Dirceu Solé"I

Cristina M A Jacob'

Departamento de Pediatria. Faculdade de Medicina. Universidade de São Paulo (Unifesp). São Paulo, SP, Brasil

" Escola Paulista de Medicina. Unifesp. São Paulo, SP, Brasil

Correspondência | Correspondence: Rejane Rimazza Dalberto Casagrande

R. Dr Enéas de Carvalho Aguiar, 647

05403-900, São Paulo, SP, Brasil

E-mail: recasag@terra.com.br

\section{Prevalência de asma e fatores de risco em escolares da cidade de São Paulo}

\section{Asthma prevalence and risk factors in schoolchildren of the city of São Paulo, Brazil}

\section{RESUMO}

OBJETIVO: Analisar a prevalência de asma e possíveis fatores de risco associados.

MÉTODOS: Estudo transversal, integrante do International Study of Asthma and Allergies in Childhood. Participaram 561 escolares de seis a sete anos de idade, provenientes de 35 escolas públicas da cidade de São Paulo, escolhidas por sorteio, em 2002. A amostra incluiu 168 asmáticos e 393 não asmáticos, que responderam questionário constituído por 33 questões referentes a dados pessoais, familiares e ambientais. A associação entre asma e fatores de risco foi avaliada pela análise de regressão logística, considerando-se nível de significância estatística de 5\%.

RESULTADOS: Entre os escolares, 31,2\% referiam sibilos nos 12 meses anteriores à entrevista. Os fatores de risco significativamente associados à asma foram: sexo masculino ( $\mathrm{OR}=2,4 ; \mathrm{IC} 95 \%$ : 1,4;4,2), mãe fumante no primeiro ano de vida $(\mathrm{OR}=2,0$; IC 95\%: 1,1;3,8), presença de eczema em locais característicos $(\mathrm{OR}=3,0 ; \mathrm{IC} 95 \%: 1,2 ; 7,6)$ e rinoconjuntivite $(\mathrm{OR}=2,4 ; \mathrm{IC}$ 95\%: 1,$2 ; 4,8)$.

CONCLUSÕES: A prevalência de asma na região estudada foi elevada e os fatores de risco relacionados foram: sexo masculino, sintomas de rinoconjuntivite no último ano, mãe fumante no primeiro ano de vida e presença de eczema em locais característicos.

DESCRITORES: Criança. Asma, epidemiologia. Fatores de risco. Estudos transversais. 


\begin{abstract}
OBJECTIVE: To assess asthma prevalence and potential risk factors associated.

METHODS: Cross-sectional study part of the International Study of Asthma and Allergies in Childhood. A total of 561 schoolchildren aged 6-7 years from 35 public schools in the city of São Paulo (Southeastern Brazil) were drawn to participate in the study, in 2002. The sample consisted of 168 asthmatic and 393 non-asthmatic children who answered a questionnaire comprising 33 questions on personal, family and environmental information. The association between asthma and the risk factors studied was assessed by logistic regression analysis at a $5 \%$ statistical significance.
\end{abstract}

RESULTS: Among the schoolchildren studied, 31.2\% reported wheezing in the 12 months preceding the interview. The following risk factors were significantly associated with asthma: male gender $(\mathrm{OR}=2.4 ; 95 \% \mathrm{CI}$ : $1.4 ; 4.2)$, maternal smoking in the child's first year of life $(\mathrm{OR}=2.0 ; 95 \% \mathrm{CI}$ : $1.1 ; 3.8)$, eczema on characteristic body areas $(\mathrm{OR}=3.0 ; 95 \% \mathrm{CI}: 1.2 ; 7.6)$ and rhinoconjunctivitis $(\mathrm{OR}=2.4 ; 95 \% \mathrm{CI}: 1.2 ; 4.8)$.

CONCLUSIONS: Asthma prevalence in the study area was high and the risk factors identified were male gender, rhinoconjunctivitis in last year, maternal smoking in the child's first year of life and eczema on characteristic body areas.

DESCRIPTORS: Child. Asthma, epidemiology. Risk Factors. Questionnaires. Cross-sectional studies.

\title{
INTRODUÇÃO
}

A asma é uma das doenças crônicas mais comuns da infância. Estudos mostram que nos últimos anos sua incidência e gravidade vêm aumentando em várias partes do mundo. ${ }^{14}$ Estudos sobre asma têm sido dificultados devido à falta de uma definição clínica para a asma que seja largamente aceita em estudos epidemiológicos e a ausência de padronização dos instrumentos utilizados para o seu diagnóstico. ${ }^{9}$

Os instrumentos mais utilizados em estudos epidemiológicos têm sido os questionários escritos auto-aplicáveis, considerados de baixo custo, fácil aplicação em grandes populações e sem influência de fatores externos como tempo, temperatura e umidade. O estudo International Study of Asthma and Allergy in Childhood (ISAAC) foi o primeiro elaborado para avaliar a prevalência de asma, rinite e eczema em duas faixas etárias pediátricas, utilizando um questionário escrito reprodutível, com estas características e tornando-se desta forma, um instrumento de aplicação universal. ${ }^{a}$

O uso do questionário escrito e padronizado do estudo ISAAC tem auxiliado a avaliação da prevalência de asma e outras doenças alérgicas desde a década de 90 , mostrando-se um método que tem permitido comparações entre diferentes regiões. ${ }^{3}$
O estudo ISAAC foi elaborado para ser realizado em três fases. A Fase I teve como objetivo a descrição da prevalência de asma, rinoconjuntivite alérgica e eczema atópico em mais de 156 centros de 56 países em duas faixas etárias (6/7 e 13/14 anos de idade). A Fase II foi projetada para investigar a importância relativa de hipóteses de interesse que surgiram da Fase I, utilizando marcadores objetivos como a broncoprovocação pela metacolina. A Fase III teve por objetivo repetir o quanto possível a metodologia aplicada em cada centro da Fase I e examinar tendências temporais na prevalência de asma, rinoconjuntivite e eczema alérgico e sua gravidade nos centros e países que participaram da Fase I do ISAAC. Além disso, o estudo ISAAC em sua Fase II tem estimulado a aplicação de um questionário complementar sobre condições ambientais, dieta e antecedentes familiares, com o objetivo de identificar possíveis fatores de risco responsáveis pela variação da prevalência de asma e outras doenças alérgicas em diferentes países ou regiões de um mesmo país.,

Para a população pediátrica no Brasil, o questionário escrito ISAAC foi validado e demonstrou sua sensibilidade e especificidade, atingindo seus objetivos em avaliar a prevalência de asma e outras doenças alérgicas. ${ }^{18} \mathrm{Na}$

${ }^{a}$ International Study of Asthma and Allergies in Childhood. ISAAC Phase One. Auckland; 1992. [acesso em: 27/02/04]. Disponível em: http://isaac.auckland.ac.nz/PhaseOne/Phs1Frame.html 
cidade de São Paulo o questionário escrito foi aplicado apenas na região centro-sul entre 1995 e 2002, mas por esta cidade ter diferentes aspectos físicos e demográficos, existia a necessidade de expandir a aplicação deste questionário em outras regiões da cidade.

A Fase I do ISAAC na faixa etária de seis a sete anos revelou prevalência de sibilos que variou de $4,1 \%$ na Indonésia a $32,1 \%$ na Costa Rica; a América Latina esteve entre as regiões de maior prevalência de asma, atingindo-se taxas médias de $19,6 \%$. $^{10, a}$

A interação entre fatores genéticos e ambientais tem sido relacionada ao aumento na prevalência de asma e outras doenças alérgicas, mas os fatores ambientais são provavelmente os maiores determinantes da manifestação dessas doenças. Vários estudos têm mostrado fatores de risco para o aumento na prevalência da asma: poluição, mudanças dietéticas, exposição alergênica e melhores condições de higiene. 1,12,16 $^{-1}$

O presente estudo teve por objetivo analisar a prevalência de asma em escolares e os fatores de risco associados.

\section{MÉTODOS}

Estudo transversal do qual participaram escolares de seis a sete anos de idade. Durante o ano de 2002 foi aplicado o questionário escrito (ISAAC) entre alunos que pertenciam a 35 escolas públicas municipais da região oeste da cidade de São Paulo, escolhidas por sorteio de um total de 42 escolas. O número total de alunos matriculados nestas 35 escolas correspondia a 5.040 alunos, dos quais $65,7 \%$ responderam ao questionário, totalizando 3.312 escolares. Os questionários foram encaminhados aos pais ou responsáveis pelos escolares, juntamente com o termo de consentimento livre e esclarecido, para preenchimento das questões, sendo orientado sua devolução em três dias.

O questionário escrito do estudo ISAAC foi previamente validado no Brasil em seus três módulos: asma, ${ }^{18}$ rinite, ${ }^{21}$ e eczema atópico. ${ }^{23} \mathrm{~A}$ opção pela participação no estudo apenas de escolas municipais, baseou-se no fato de os alunos estarem vinculados a endereços próximos das escolas, incluindo famílias moradoras de núcleos com condições de vida precária (favelas), o que poderia evidenciar menor percentual de doenças alérgicas baseado na hipótese da higiene.

A questão sobre a presença de sibilos nos últimos 12 meses foi utilizada para a avaliação da prevalência de asma por reduzir os erros de memória e impedir a interferência de fatores sazonais no mês de aplicação do questionário. ${ }^{\text {a }}$ Foram denominados asmáticos, os escolares que apresentaram resposta positiva, e não asmáticos, os que apresentaram resposta negativa a essa questão.

O questionário complementar da Fase II do estudo ISAAC, foi constituído por 33 questões referentes a dados pessoais, familiares e ambientais, tendo a finalidade de avaliar a associação entre possíveis fatores de risco e o desenvolvimento de asma. ${ }^{b}$ Foram também incluídos fatores de risco, a presença de outras doenças alérgicas como rinite, rinoconjuntivite, dermatite atópica e presença de eczema em locais característicos, avaliados pelo questionário escrito ISAAC.

O questionário complementar foi aplicado a escolares participantes da Fase III do estudo ISAAC, provenientes de escolas que preenchiam os seguintes critérios: número de alunos participantes maior que 100/escola e percentual de devolução do questionário escrito maior ou igual a $70 \%$, totalizando o envio do questionário complementar a 827 alunos.

Para o cálculo da amostra dos subgrupos asma e não-asma que foram submetidos ao questionário complementar, utilizou-se o programa Instat 3/Statmate. Considerando-se um estudo de caso-controle, foram selecionados dois controles para cada caso, supondo que a prevalência para cada fator de risco relacionados à asma seria de $20 \%$ no grupo controle, com odds ratio $(\mathrm{OR})$ de 1,5 , erro alfa de $5 \%$ e poder de teste de $85 \%$. Dessa forma, a amostra necessária foi definida por 120 casos (asmáticos) para 240 controles (não asmáticos). Como o percentual de devolução de questionário escrito foi de $65,7 \%$, considerou-se necessário o envio de questionário complementar a uma amostra inicial que pudesse compensar as possíveis perdas e assegurar a amostra final desejada. A amostra final constituiu-se de 561 escolares, com 168 asmáticos e 393 não-asmáticos.

Diferenças nas proporções foram avaliadas utilizandose o teste exato de Fisher. Em relação aos dados do questionário complementar, os grupos de asmáticos e não asmáticos foram comparados em relação aos diversos fatores de risco. O programa SPSS 10.0 foi utilizado para o cálculo do OR, intervalo de confiança de 95\% (IC 95\%) e obtenção das análises univariada e multivariada. Os fatores de risco analisados estão mostrados na Tabela 1, e aqueles que apresentavam valores de $\mathrm{p}<0,20$ na análise univariada foram incluídos no modelo de regressão logística (Bakward stepwise wald). ${ }^{6} \mathrm{Na}$ análise multivariada, os valores de $\mathrm{p}<0,05$ foram considerados de significância estatística.

\footnotetext{
a International Study of Asthma and Allergies in Childhood. ISAAC Phase Two. Auckland; 1992. [acesso em: 27/02/04]. Disponível em: http://isaac.auckland.ac.nz/Phasetwo/Phs2Frame.html

b International Study of Asthma and Allergies in Childhood. ISAAC Phase Two. Auckland; 1992. [acesso em: 27/02/04]. Disponível em: http:// isaac.auckland.ac.nz/Phasetwo/Phs2Frame.html
} 
Os pais ou responsáveis pelos escolares assinaram termo de consentimento livre esclarecido. O projeto de pesquisa foi aprovado pelo Comitê de Ética e Pesquisa do Departamento de Pediatria e pela Comissão de Ética para Análise de Projetos de Pesquisa do Hospital das Clínicas da Faculdade de Medicina da Universidade de São Paulo.

\section{RESULTADOS}

Na amostra inicial de 3.312 escolares que responderam ao questionário escrito houve uma predominância do sexo feminino $(50,6 \%$ vs $49,4 \%)$ e de escolares com seis anos de idade $(59,7 \%$ vs $40,3 \%)$.
O número total de escolares que apresentaram sibilância alguma vez na vida foi de $55,2 \%(\mathrm{~N}=1.829)$, enquanto $31,2 \%(\mathrm{~N}=1.033)$ responderam positivamente à questão sobre presença de sibilos nos últimos 12 meses, sendo considerados asmáticos.

O questionário complementar foi distribuído a 827 escolares pertencentes a seis escolas participantes do estudo, com retorno de 561 questionários $(67,8 \%)$ corretamente preenchidos e devolvidos, que constituíram a amostra para avaliação dos fatores de risco. Entre eles, 168 foram considerados asmáticos e 393 não asmáticos, com predomínio do sexo feminino $(51,2 \%$ vs $48,8 \%)$.

Tabela 1. Análise univariada dos fatores de risco para presença ou não de asma. Município de São Paulo, SP, 2002.

\begin{tabular}{|c|c|c|c|c|c|}
\hline Fator de risco & $\begin{array}{l}\text { OR não } \\
\text { ajustado }\end{array}$ & IC $95 \%$ & $\mathrm{p}$ & $\begin{array}{c}\text { Asmáticos } \\
\text { expostos (\%) }\end{array}$ & $\mathrm{N}$ \\
\hline Presença de gato dentro de casa atualmente & 1,0 & 0,$6 ; 1,9$ & 1,000 & $17(3)$ & 561 \\
\hline Mãe fumante & 1,0 & 0,$7 ; 1,5$ & 0,910 & $41(8)$ & 513 \\
\hline Idade de início em creche, berçário ou jardim infância > 1 ano & 1,0 & 0,$6 ; 1,6$ & 0,890 & $86(23)$ & 378 \\
\hline Pai atópico & 1,1 & 0,$6 ; 1,9$ & 0,780 & $21(4)$ & 561 \\
\hline Presença de gato dentro de casa no primeiro ano de vida & 0,8 & 0,$4 ; 1,8$ & 0,690 & $8(1)$ & 561 \\
\hline Não admissão em creche, berçário ou jardim infância & 1,1 & 0,$7 ; 1,6$ & 0,680 & $49(9)$ & 558 \\
\hline Presença de refrigerante na dieta de 3 a 6 vezes semana & 1,6 & 0,$5 ; 5,0$ & 0,600 & $57(26)$ & 220 \\
\hline Parto cesáreo & 0,9 & 0,$6 ; 1,3$ & 0,56 & $62(12)$ & 513 \\
\hline Verminose & 1,2 & 0,$7 ; 2,2$ & 0,54 & $19(4)$ & 453 \\
\hline Não ter irmão mais velho & 1,1 & 0,$8 ; 1,7$ & 0,51 & $69(12)$ & 559 \\
\hline Mãe fumante durante gravidez & 1,2 & 0,$7 ; 2,1$ & 0,47 & $24(7)$ & 359 \\
\hline Presença de cão dentro de casa atualmente & 1,2 & 0,$8 ; 1,8$ & 0,41 & $54(10)$ & 561 \\
\hline Presença de animais dentro de casa no primeiro ano de vida & 1,2 & 0,$8 ; 1,8$ & 0,34 & $49(9)$ & 561 \\
\hline Fumante dentro de casa & 1,2 & 0,$8 ; 1,8$ & 0,33 & $65(12)$ & 550 \\
\hline Ausência de frutas frescas na dieta & 0,5 & 0,$2 ; 1,6$ & 0,31 & $4(3)$ & 133 \\
\hline Presença de animais dentro de casa atualmente & 1,2 & 0,$8 ; 1,7$ & 0,30 & $78(14)$ & 561 \\
\hline Irmãos gêmeos & 1,8 & 0,$7 ; 4,5$ & 0,30 & $8(1)$ & 552 \\
\hline Ausência de vegetais crus na dieta & 1,5 & 0,$7 ; 3,1$ & 0,29 & $41(21)$ & 194 \\
\hline Ausência de doença sarampo & 0,7 & 0,$4 ; 1,2$ & 0,22 & $126(25)$ & 495 \\
\hline Ausência de peixe na dieta & 1,5 & 0,$8 ; 2,8$ & 0,21 & $27(13)$ & 205 \\
\hline Sexo masculino & 1,3 & 0,$9 ; 1,9$ & 0,16 & $90(16)$ & 561 \\
\hline Peso nascimento $<2500 \mathrm{~g}$ & 1,4 & 0,$9 ; 2,3$ & 0,16 & $32(6)$ & 525 \\
\hline Aleitamento materno $<6$ meses & 1,3 & 0,$9 ; 2,0$ & 0,12 & $64(14)$ & 474 \\
\hline Mãe fumante no $1^{\circ}$ ano de vida & 1,5 & 0,$9 ; 2,6$ & 0,11 & $28(8)$ & 345 \\
\hline Presença de cão dentro de casa no primeiro ano de vida & 1,5 & 1,$0 ; 2,4$ & 0,045 & $40(7)$ & 561 \\
\hline Presença de eczema em locais característicos & 3,0 & 1,$7 ; 5,7$ & $<0,001$ & $25(4)$ & 561 \\
\hline Ausência de divisão do quarto no primeiro ano de vida & 0,1 & 0,$02 ; 0,5$ & 0,001 & $2(1)$ & 339 \\
\hline Dermatite atópica & 3,0 & 1,$7 ; 5,0$ & $<0,001$ & $33(6)$ & 561 \\
\hline Rinoconjuntivite & 3,1 & 1,$9 ; 5,0$ & $<0,001$ & $43(8)$ & 561 \\
\hline Rinite isolada & 1,9 & 1,$2 ; 3,0$ & 0,005 & $42(7)$ & 561 \\
\hline Mãe atópica & 2,4 & 1,$6 ; 3,6$ & $<0,001$ & $67(12)$ & 561 \\
\hline Umidade na casa & 2,2 & 1,$5 ; 3,3$ & $<0,001$ & $66(12)$ & 549 \\
\hline
\end{tabular}


A Tabela 2 mostra todos os fatores de risco incluídos na análise multivariada, utilizando como variável dependente asma (positividade da questão 2 sobre presença de sibilos nos últimos 12 meses), com respectivos OR, IC 95\%, nível de significância (p), percentagem de asmáticos expostos e amostra total de cada fator.

Tabela 2. Análise multivariada dos fatores de risco para asma. Município de São Paulo, SP, 2002.

\begin{tabular}{lccccc}
\hline $\begin{array}{l}\text { Fator de } \\
\text { risco }\end{array}$ & $\begin{array}{c}\text { Asma } \\
(\%)\end{array}$ & $\begin{array}{c}\text { OR não } \\
\text { ajustado }\end{array}$ & $\begin{array}{c}\text { OR } \\
\text { Ajustado }\end{array}$ & $\mathrm{p}$ & $\mathrm{N}$ \\
& & (IC 95\%) & $($ IC 95\%) & & \\
\hline
\end{tabular}

Mãe atópica

$\begin{array}{ccccc}\text { Sim } & 12 & 2,4 & 1,3 & <0,001 \\ \text { Não } & 15 & (1,6 ; 3,6) & (0,7-2,4) & \end{array}$

Aleitamento materno $<6$ meses

$\begin{array}{ccccc}\text { Sim } & 14 & 1,3 & 1,3 & 0,39 \\ \text { Não } & 16 & (0,9 ; 2,0) & (0,7 ; 2,2) & \end{array}$

Peso ao nascimento $<2.500 \mathrm{~g}$

$\begin{array}{cccccr}\text { Sim } & 6 & 1,4 & 1,3 & 0,57 & \\ \text { Não } & 24 & (0,9 ; 2,3) & (0,6 ; 2,8) & & 525\end{array}$

Presença de cão no domicílio no primeiro ano de vida
Sim
1,5
1,6
0,01
Não
$23 \quad(1,0 ; 2,4)$
$(0,9 ; 3,0)$

Eczema*

Sim

$$
\begin{array}{cc}
6 & 3,0 \\
24 & (1,7 ; 5,0)
\end{array}
$$$$
1,6
$$$$
0,39
$$$$
24
$$

Umidade no domicílio

$\begin{array}{ll}\text { Sim } & 12 \\ \text { Não } & 18\end{array}$

$$
2,2
$$$$
(0,5 ; 4,6)
$$

Rinite** $^{* *}$

$\begin{array}{ccc}\text { Sim } & 7 & 1,9 \\ \text { Não } & 22 & (1, \\ & & 2 ; 3,0)\end{array}$

$$
\begin{gathered}
1,8 \\
(0,9 ; 3,6)
\end{gathered}
$$

Mãe fumante no primeiro ano de vida

$\begin{array}{cccccc}\text { Sim } & 8 & 1,5 & 2,0 & 0,03 & 345 \\ \text { Não } & 23 & (0,9 ; 2,6) & (1,1 ; 3,8) & & \end{array}$

Sexo masculino

$\begin{array}{cccccc}\text { Sim } & 16 & 1,3 & 2,4 & 0,002 & 561 \\ \text { Não } & 14 & (0,9 ; 1,9) & (1,4 ; 4,2) & & \end{array}$

Rinoconjuntivite***

$\begin{array}{cccccc}\text { Sim } & 8 & 3,1 & 2,4 & 0,012 & 561 \\ \text { Não } & 22 & (1,9 ; 5,0) & (1,2 ; 4,8) & & \end{array}$

Eczema atópico em locais característicos****

\begin{tabular}{cccccc} 
Sim & 4 & 3,0 & 3,0 & 0,018 & 561 \\
Não & 25 & $(1,7 ; 5,7)$ & $(1,2 ; 7,6)$ & & \\
\hline
\end{tabular}

* Questão 2 - questionário escrito ISAAC - módulo eczema ** Questão 2 - questionário escrito ISAAC - módulo rintite

*** Questão 3 - questionário escrito ISAAC - módulo rintite **** Questão 3 - questionário escrito ISAAC - módulo eczema
Após a análise multivariada, quatro fatores permaneceram significantes: mãe fumante no primeiro ano de vida $(\mathrm{OR}=2,0$; IC 95\%: 1,1;3,8; $\mathrm{p}=0,031)$; rinoconjuntivite (OR=2,4; IC 95\%: 1,2;4,8; $\mathrm{p}=0,012$ ); sexo masculino (OR=2,4; IC 95\%: 1,4;4,2; $\mathrm{p}=0,002)$; eczema em locais característicos $(\mathrm{OR}=3,0 ; \mathrm{IC} 95 \%: 1,2 ; 7,6 ; \mathrm{p}=0,018)$.

\section{DISCUSSÃO}

No presente estudo a prevalência da asma em $31,2 \%$ dos escolares (resposta afirmativa à questão sobre sibilância no último ano), na região oeste de São Paulo foi maior que o encontrado na região centro-sul de São Paulo $(24,4 \%)$ e em todos os outros centros participantes da Fase III no Brasil. ${ }^{19} \mathrm{~A}$ mesma tendência foi observada em relação aos dados da América Latina e dados mundiais, onde a média encontrada foi de $19,6 \%$ e $10,2 \%$ respectivamente, sugerindo que a região oeste da cidade de São Paulo apresenta as maiores prevalências encontradas nesta faixa etária. ${ }^{10}$ Uma das hipóteses para o encontro da alta prevalência de asma nesta população foi o seu baixo nível socioeconômico, já descrito por Mallol na fase I do estudo ISAAC na América Latina. ${ }^{10}$

Alguns fatores ambientais atuando no início da vida e sua interação com genes específicos para atopia poderiam ser os responsáveis pelo desenvolvimento da asma, uma doença crônica e persistente. ${ }^{1}$

Os fatores genéticos, apesar de serem importantes, não conseguem explicar as diferenças na prevalência da asma nos diversos continentes, nem o seu aumento nos últimos 40 anos, por ser um período muito curto para que alguma mudança genética tenha ocorrido. ${ }^{22} \mathrm{Assim}$, os fatores ambientais como mudanças no estilo de vida, dieta e aumento da exposição aos alérgenos intradomiciliares têm sido relacionados como potenciais determinantes da maior prevalência das doenças alérgicas. ${ }^{1}$

No presente estudo, a rinoconjuntivite foi considerada como fator de risco para asma e conhecimentos atuais ${ }^{4}$ relatam que a presença de sintomas nasais associados aos oculares confere maior sensibilidade ao diagnóstico de rinite alérgica.

Em relação ao eczema, a variável de maior significância para o desenvolvimento da asma foi a presença de eczema em locais típicos. Yamada et $a^{23}$ observaram sensibilidade de $92 \%$ e especificidade de $96 \%$ para esse sintoma durante a validação do questionário escrito ISAAC para eczema atópico.

É clara a relação entre aparecimento precoce de eczema atópico e rinite precedendo o desenvolvimento de asma, e o termo "marcha atópica" é utilizado para descrever tal fenômeno. ${ }^{20}$ Os resultados no presente estudo, após análise multivariada, mostraram correlação entre doenças alérgicas e asma, mostrando a necessidade da busca de outras doenças atópicas na avaliação global do paciente asmático. 
Durante toda a infância, ser do sexo masculino é um fator de risco para asma e na adolescência esta tendência se inverte. O presente estudo encontrou 2,4 vezes maior risco para asma no sexo masculino. Uma das hipóteses para justificar este predomínio seria o menor diâmetro e maior tônus das vias aéreas, com menores fluxos pulmonares durante o primeiro ano de vida mais evidente em meninos do que em meninas. ${ }^{5}$

A exposição passiva à fumaça de cigarro tem sido relacionada à doença sibilante nos primeiros anos de vida e a asma na idade escolar. Estudo longitudinal desenvolvido por Martinez et $\mathrm{l}^{11}$ mostrou que crianças cujas mães fumavam mais de meio maço de cigarros por dia, principalmente nos primeiros dois anos de vida, tiveram cerca de duas vezes mais chance de desenvolver asma. Os resultados da presente casuística mostrou que escolares com mães fumantes no primeiro ano de vida têm risco duas vezes maior de desenvolver asma, podendo este fato estar associado ao maior contato da mãe com a criança nesta fase da infância.

Estudos têm mostrado a importância da mãe atópica como fator de risco para asma, provavelmente relacionada à maior passagem de citocinas, antígenos e/ou anticorpos através da placenta. ${ }^{2}$ Nesta avaliação, embora a presença de mãe atópica tenha sido significante na análise univariada, este fator não se mostrou significante após a análise multivariada.

Foram incluídos na análise multivariada fatores de risco que a literatura classicamente refere como relevantes para o desenvolvimento da asma e que apresentassem valor de $\mathrm{p}<0,2$ na análise univariada. Outros fatores de risco de menor importância demandariam uma amostra mais expressiva para se manter pelo menos $80 \%$ de poder do estudo.

Em relação ao aleitamento materno, estudos transversais e longitudinais têm relatado seu efeito protetor para asma, ${ }^{8}$ enquanto outros descrevem-no como fator de risco a esta doença. ${ }^{17}$ No presente estudo, o aleitamento materno por um período mínimo de seis meses não apresentou significância após a análise multivariada.

Chatkin \& Menezes ${ }^{7}$ encontraram correlação entre baixo peso de nascimento como fator de risco para asma, especialmente entre crianças até cinco anos de idade, associação não confirmada no presente estudo após análise multivariada.

A "hipótese da higiene" relaciona a menor exposição a microorganismos como fator de risco para desenvolvimento de doenças alérgicas e tem sido extensamente discutida na literatura. ${ }^{15}$ De modo geral, a hipótese da higiene pode explicar o aumento na prevalência de atopia em populações com melhores condições socioeconômicas e ambientais, mas em relação a asma as evidências parecem ser menos convincentes. A presente casuística não encontrou associação entre asma e os fatores de risco relacionados à hipótese da higiene como: presença de cão ou animais no domicílio, presença de irmãos mais velhos na casa, infecções comuns na infância, dieta e umidade. Para testar essa hipótese, a população de escolares analisadas no presente estudo foi composta por maioria proveniente de classes econômicas menos favorecidas e moradores em comunidades faveladas.

Em estudo semelhante desenvolvido em adolescentes (13 e 14 anos) na mesma região utilizando a metodologia ISAAC, Pastorino et $\mathrm{al}^{13}$ encontraram como fatores de risco nesta faixa etária: prematuridade $(\mathrm{OR}=3,84)$, rinite $(\mathrm{OR}=3,18)$, eczema em locais característicos $(\mathrm{OR}=2,86)$, positividade para o teste cutâneo de hipersensibilidade imediata para aeroalérgenos $(\mathrm{OR}=2,81)$, mãe atópica $(\mathrm{OR}=2,01)$ e como fator de proteção o consumo de vegetais cozidos $(\mathrm{OR}=0,37)$.

Estudos transversais possuem como limitação o tempo e a cronologia em relação à exposição aos fatores de risco. Apesar dessa limitação, eles têm sido utilizados para o reconhecimento de possíveis causas a serem incluídas em estudos de coorte, que são mais adequados para descrever os fatores de risco de uma doença. A inclusão de escolares com baixa condição socioeconômica e ambiental pode ter trazido um viés ao estudo, pois foi analisado um subgrupo da região oeste da cidade de São Paulo. Novos estudos aplicando a mesma metodologia em alunos de escolas particulares e estaduais poderiam complementar dados sobre fatores de risco ou mesmo sobre a prevalência de asma nesta região da cidade de São Paulo.

O presente estudo, utilizando a metodologia ISAAC na faixa etária de seis a sete anos, permitiu o conhecimento da prevalência de asma e fatores de risco relacionados na região oeste da cidade de São Paulo. Estes resultados poderão ser utilizados em futuros estudos epidemiológicos, objetivando um conhecimento mais abrangente da evolução da asma e a compreensão dos fenômenos biológicos relacionados, permitindo elaboração de medidas de prevenção eficazes para o controle desta doença. Em especial no Brasil, essas pesquisas se revelam importantes, pelo desafio da coexistência entre alta prevalência de asma e precárias condições socioeconômicas, muitas vezes contrariando a hipótese da higiene. 


\section{REFERÊNCIAS}

1. Arruda LK, Sole D, Baena-Cagnani CE, Naspitz CK. Risk factors for asthma and atopy. Curr Opin Allergy Clin Immunol. 2005;5(2):153-9.

2. Arshad $\mathrm{SH}$. Development of allergic disease in children. Clin Exp Allergy. 1997;27(11):1231-3.

3. Asher MI, Keil U, Anderson HR, Beasley R, Crane J, Martinez F, et al. International Study of Asthma and Allergies in Childhood (ISAAC): Rationale and methods. Eur Respir J. 1995;8(3):483-91.

4. Bousquet J, Van Cauwenberge P, Khaltaev N. Allergic rhinitis and its impact on asthma. J Allergy Clin Immunol. 2001;108(5 suppl):S147-S334.

5. Caracta CF. Gender differences in pulmonary disease. Mt Sinai J Med. 2003;70(4):215-24

6. Chan YH. Biostatistics 202: logistic regression analysis. Singapore Med J. 2004;45(4):149-53.

7. Chatkin MN, Menezes AM. Associação entre baixo peso ao nascer e asma: uma revisão sistemática da literatura. Rev Panam Salud Publica. 2005;17(2):102-9.

8. Friedman NJ, Zeiger RS. The role of breast-feeding in the development of allergies and asthma. J Allergy Clin Immunol. 2005;115(6):1238-48.

9. Magnus P, Jaakkola JJ. Secular trend in the occurrence of asthma among children and young adults: critical appraisal of repeated cross sectional surveys. BMJ. 1997;314(7097):1795-9.

10. Mallol J, Solé D, Asher I, Clayton T, Stein R, SotoQuiroz M. Prevalence of asthma symptoms in Latin America: the International Study of Asthma and Allergies in Childhood (ISAAC). Pediatr Pulmonol. 2000;30(6):439-44.

11. Martinez FD, Cline M, Burrows B. Increased incidence of asthma in children of smoking mothers. Pediatrics. 1992;89(1):21-6.

12. Matricardi PM. Prevalence of atopy and asthma in eastern versus western Europe: why the difference? Ann Allergy Asthma Immunol. 2001;87(6 Supl 3):24-7.

13. Pastorino AC, Rimazza RD, Leone C, Castro AP, Sole D, Jacob CM. Risk factors for asthma in adolescents in large urban region of Brazil. J Asthma. 2006;43(9):695700 .
14. Phelan PD. Asthma in children: epidemiology. BMJ. 1994;308(6944):1584-5.

15. Platts-Mills TA, Erwin E, Heymann P, Woodfolk J. Is the hygiene hypothesis still a viable explanation for the increased prevalence of asthma? Allergy. 2005;60(Supl 79):25-31

16. Sears MR. Epidemiology of childhood asthma. Lancet. 1997;350(9083):1015-20.

17. Sears MR, Greene JM, Willan AR, Taylor DR, Flannery EM, Cowan JO, et al. Long-term relation between breastfeeding and development of atopy and asthma in children and young adults: a longitudinal study. Lancet. 2002;360(9337):901-7.

18. Solé D, Vanna AT, Yamanda E, Rizzo MC, Naspitz CK. International Study of Asthma and Allergies in Childhood (ISAAC) written questionnaire: validation of the asthma component among Brazilian children. J Invest Allergol Clin Immunol. 1998;8(6):376-82.

19. Solé D, Wandalsen GF, Camelo-Nunes IC, Naspitz CK, ISAAC - Brazilian Group. Prevalence of symptoms of asthma, rhinitis and atopic eczema among Brazilian children and adolescents identified by the International Study of Asthma and Allergies in Childhood (ISAAC) - Phase 3. J Pediatr (Rio J). 2006;82(5):341-6.

20. Spergel JM. Atopic march: link to upper airways. Curr Opin Allergy Clin Immunol. 2005;5(1):17-21.

21. Vanna AT, Yamada E, Arruda LK, Naspitz CK, Solé D. International Study of Asthma and Allergies in Childhood: Validation of the rhinitis symptom questionnaire and prevalence of rhinitis in schoolchildren in São Paulo, Brazil. Pediatr Allergy Immunol. 2001;12(2):95-101.

22. Von Mutius E. The environmental predictors of allergic disease. J Allergy Clin Immunol. 2000;105(1 Pt 1):9-19.

23. Yamada E, Vanna AT, Naspitz CK, Solé D. International Study of Asthma and Allergies in Childhood: validation of the written questionnaire (eczema component) and prevalence of atopic eczema among Brazilian children. J Invest Allergol Clin Immunol. 2002;12(1):34-41.

Artigo baseado em dissertação de mestrado de RRD Casagrande apresentada à Faculdade de Medicina da Universidade de São Paulo, em 2006. 